\title{
High Cytoplasmic CXCR4 Expression Predicts Prolonged Survival in Triple-Negative Breast Cancer Patients Treated with Adjuvant Chemotherapy
}

\author{
Bobae Shim · Min-Sun Jin ${ }^{1}$ \\ Ji Hye Moon · In Ae Park \\ Han Suk Ryu
}

Department of Pathology, Seoul National University Hospital, Seoul National University College of Medicine, Seoul; ' ${ }^{1}$ epartment of Pathology, Bucheon St. Mary's Hospital, College of Medicine, The Catholic University of Korea, Bucheon, Korea

Received: August 7, 2018

Revised: September 12, 2018

Accepted: September 18, 2018

Corresponding Author

Han Suk Ryu, MD, PhD

Department of Pathology, Seoul National University Hospital, Seoul National University College of

Medicine, 101 Daehak-ro, Jongno-gu,

Seoul 03080, Korea

Tel: +82-2-2072-3361

Fax: $+82-2-743-5530$

E-mail: karlnash@naver.com
Background: Chemokine receptor CXC chemokine receptor type 4 (CXCR4) and its ligand CXC motif chemokine 12 (CXCL12; stromal cell-derived factor-1) are implicated in tumor growth, metastasis, and tumor cell-microenvironment interaction. A number of studies have reported that increased CXCR4 expression is associated with worse prognosis in triple-negative breast cancer (TNBC), but its prognostic significance has not been studied in TNBC patients treated with adjuvant chemotherapy. Methods: Two hundred eighty-three TNBC patients who received adjuvant chemotherapy were retrospectively analyzed. Tissue microarray was constructed from formalinfixed, paraffin-embedded tumor tissue and immunohistochemistry for CXCR4 and CXCL12 was performed. Expression of each marker was compared with clinicopathologic characteristics and outcome. Results: High cytoplasmic CXCR4 expression was associated with younger age $(p=$ $.008)$, higher histologic grade $(p=.007)$ and lower pathologic stage $(p=.045)$, while high CXCL12 expression was related to larger tumor size $(p=.045)$, positive lymph node metastasis $(p=.005)$, and higher pathologic stage $(p=.017)$. The patients with high cytoplasmic CXCR4 experienced lower distant recurrence $(p=.006)$ and better recurrence-free survival (RFS) (log-rank $p=.020)$ after adjuvant chemotherapy. Cytoplasmic CXCR4 expression remained an independent factor of distant recurrence $(p=.019)$ and RFS $(p=.038)$ after multivariate analysis. Conclusions: High cytoplasmic CXCR4 expression was associated with lower distant recurrence and better RFS in TNBC patients treated with adjuvant chemotherapy. This is the first study to correlate high CXCR4 expression to better TNBC prognosis, and the underlying mechanism needs to be elucidated in further studies.

Key Words: CXCR4; CXCL12; Triple negative breast neoplasms; Prognostic marker
Triple-negative breast cancer (TNBC) refers to the breast cancer subtype which does not express estrogen receptor (ER), progesterone receptor (PR), and lacks overexpression of human epidermal growth factor receptor 2 (HER2). It comprises 10\%-20\% of all breast cancer cases and is associated with aggressive behavior and poor prognosis. ${ }^{1}$ TNBC is generally considered an individual subtype of breast cancer, but it is also a highly heterogeneous disease which consists of various subgroups of tumors with different molecular, histologic, and clinical characteristics. ${ }^{2}$ Advances in endocrine therapy and HER2-targeted therapy have greatly improved the survival of the patients with hormone receptor-positive and HER2-positive tumors, but TNBC patients still suffer from absence of specific treatment target. Systemic chemotherapy continues to be the mainstay of TNBC treatment, and there is an urgent need for novel biomarkers which can be used to predict prognosis, identify patients who will benefit from therapy, and provide potential treatment target. ${ }^{2}$
CXC chemokine receptor type 4 (CXCR4) is a member of $\mathrm{G}$ protein-coupled receptors which is bound by its only ligand CXC motif chemokine 12 (CXCL12), also known as stromal cell-derived factor- $1{ }^{3}$ It is physiologically involved in embryonic development, leukocyte trafficking and homing of hematopoietic cells to bone marrow. ${ }^{4-6}$ In tumor biology, the CXCR 4/CXCL12 axis is known to promote proliferation of tumor cells, direct metastasis by attracting CXCR4-positive tumor cells to CXCL12-rich organs, and mediate the interaction between the tumor cell and their microenvironment. ${ }^{7-9}$ CXCR 4 is expressed in different cancer types, and its overexpression and association with distant metastasis and unfavorable prognosis have been reported in breast cancer. $^{3,9-11}$ In addition, targeting of CXCR 4 significantly reduced both primary and metastatic breast cancer in the mouse model, suggesting that CXCR4/CXCL12 axis may be a promising therapeutic target in breast cancer treatment. ${ }^{12,13}$

A number of studies have reported the negative prognostic sig- 
nificance of CXCR 4 expression in TNBC, but adjuvant treatment information is not clearly documented in most of these reports, leaving the possibility of confounding. ${ }^{14-16}$ Moreover, although systemic chemotherapy is an essential element of TNBC treatment, the prognostic significance of CXCR 4 has not been studied in TNBC patients treated with adjuvant chemotherapy. Therefore, we aimed to evaluate the expression of CXCR 4 in TNBC tumor tissue, compare it with clinicopathologic parameters, and investigate its relationship with the outcome of the patients who received adjuvant chemotherapy. Since the expression of CXCL12 has not been well-addressed in TNBC, we planned to evaluate its expression as well.

\section{MATERIALS AND METHODS}

\section{Patients and tissue samples}

The study group consisted of primary unilateral TNBC patients who underwent surgical resection in Seoul National University Hospital between December 2000 and December 2006 and received adjuvant chemotherapy. The cases with available formalin-fixed, paraffin-embedded (FFPE) tissue for tissue microarray (TMA) were retrospectively collected. The patients who had distant metastasis at initial diagnosis, received neoadjuvant chemotherapy, underwent surgical resection for bilateral breast cancer, or had a history of ipsilateral or contralateral breast cancer were excluded from the study. Immunohistochemistry (IHC) for ER, PR, and HER2 was routinely performed on resection specimen at the time of diagnosis, and the IHC slides were reviewed. According to the American Society of Clinical Oncology/College of American Pathologists (ASCO/CAP) guidelines, ER and PR negativity was defined as nuclear staining in $<1 \%$ of tumor cells in IHC, and HER2 was considered negative if a tumor was scored 0 or $1+$ in HER2 IHC or $2+$ with a negative HER2 fluorescence in situ hybridization result. ${ }^{17,18}$ Among the 313 primary TNBC patients identified, 283 patients $(90.4 \%)$ received adjuvant chemotherapy. The patients were treated with standard chemotherapy and the regimens were classified into three categories: anthracycline-based regimen, taxane-anthracycline-based regimen and CMF (cyclophosphamide, methotrexate, and 5-fluorouracil). Use of taxane-anthracycline-based regimen was limited to node-positive tumors due to insurance constraints. More detailed information on chemotherapy regimen is shown in Table 1.

Clinicopathologic characteristics, treatment details, and follow-up data were retrieved from medical records and the original pathology report. Histologic grade was scored according to the Nottingham grading system and the pathologic stage was
Table 1. Chemotherapy regimen in patients treated with adjuvant chemotherapy

\begin{tabular}{lc}
\hline Adjuvant chemotherapy regimen & No. of patients \\
\hline Anthracycline-based & 164 \\
FAC & 132 \\
AC & 10 \\
FEC & 21 \\
EC & 1 \\
Taxane-anthracycline-based & 51 \\
AC $\rightarrow$ paclitaxel and/or docetaxel & 50 \\
FEC $\rightarrow$ paclitaxel & 1 \\
CMF & 65 \\
Change of regimen & $1^{\text {a }}$ \\
Unknown & 2 \\
Total & 283 \\
\hline
\end{tabular}

FAC, 5-fluorouracil (5-FU), doxorubicin, and cyclophosphamide; AC, doxorubicin and cyclophosphamide; FEC, 5-FU, epirubicin, and cyclophosphamide; EC, epirubicin and cyclophosphamide; CMF, cyclophosphamide, methotrexate, and 5-FU.

${ }^{\mathrm{A} A C}$ to CMF.

determined based on the American Joint Committee on Cancer staging system, seventh edition. ${ }^{19}$ Follow-up and survival data were collected until the end of 2014. The date of recurrence, death, and last follow-up were obtained from medical records, and recurrence-free survival (RFS) and overall survival (OS) were assessed according to STEEP criteria. ${ }^{20}$ Recurrence was diagnosed either pathologically or radiologically and was classified as locoregional or distant. This study was approved by the Institutional Review Board of Seoul National University Hospital with a waiver of informed consent (IRB No. 1512-076-728).

\section{TMA construction and IHC}

Hematoxylin and eosin-stained slides of each tumor were reviewed and the representative area was marked. Cylindrical tissue core with a diameter of $2 \mathrm{~mm}$ was extracted from the corresponding area of the FFPE tumor block and transferred into recipient paraffin block (SuperBioChips Laboratories, Seoul, Korea). Each TMA block included a maximum of 59 cores. TMA blocks were sectioned at the 3- $\mu \mathrm{m}$ thickness and IHC for CXCR 4 and CXCL12 was performed with automated staining system (BenchMark XT, Ventana Medical Systems, Tucson, AZ, USA) following the manufacturer's protocols. TMA sections were first deparaffinized, and antigen retrieval was done using cell conditioning solution (CC1, Ventana Medical Systems). Then sections were incubated with primary rabbit polyclonal anti-CXCR 4 (1:50, ab2074, Abcam, Cambridge, UK) and mouse monoclonal antiCXCL12 (1:10, MAB350, R\&D Systems, Minneapolis, MN, USA) antibodies. The positive antigen-antibody reaction was visualized using diaminobenzidine detection kit (OptiView DAB, 
Ventana Medical Systems), and counterstaining was performed with hematoxylin and bluing reagent.

The IHC slides were examined blindly without knowledge of clinicopathologic information, and the expression of CXCR 4 and CXCL12 was assessed by IHC using a semiquantitative scoring system. The staining intensity was scored as 0 (negative), 1 (weak), 2 (moderate), and 3 (strong), and percentage of positively stained cells were scored as $0(0 \%), 1(1 \%-25 \%), 2$ (26\%$50 \%), 3(51 \%-75 \%)$, and $4(>75 \%)$. The final score was calculated by multiplying the intensity and percentage scores, ranging from 0 to 12. The patients were divided into high or low expression groups using the median score of each marker as a cutoff point.

\section{Statistical analysis}

Differences in clinicopathologic variables and outcomes between high and low expression groups were compared using the chi-square test, or Fischer exact test when applicable. Survival curves were generated using the Kaplan-Meier method and com- pared using the log-rank test. Cox proportional hazards model and logistic regression analysis were used to evaluate the prognostic significance of each variable in the univariate and multivariate analysis. Variables with a p-value of $<.20$ in univariate analysis were included in multivariate analysis, and forward conditional method was used to select the significant variables. All statistical analyses were performed using SPSS Statistics software ver. 22.0 (IBM Corp., Armonk, NY, USA), and a p-value of <.05 was considered statistically significant.

\section{RESULTS}

\section{Expression of CXCR4 and CXCL12 in TNBC tissues}

IHC for CXCR 4 and CXCL12 was performed on TMA section, and due to core loss and noninformative cores with no invasive carcinoma, expression of CXCR 4 and CXCL12 were evaluable in 259 (91.5\%) and 238 (84.1\%) cases, respectively. Immunostaining for CXCR 4 was observed in tumor cells, stromal cells,
Staining intensity

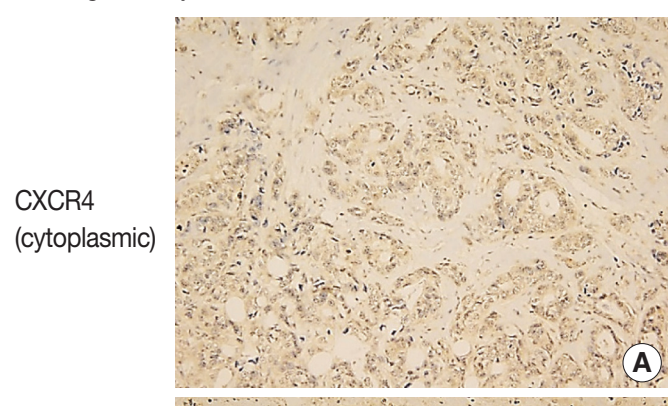

2

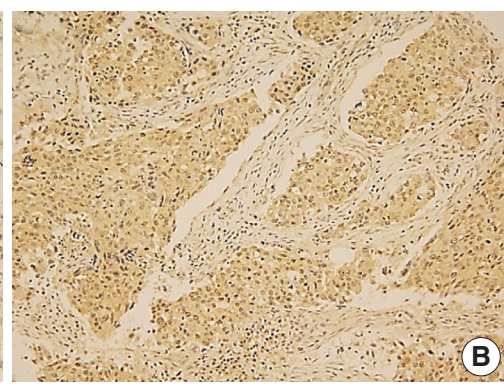

3

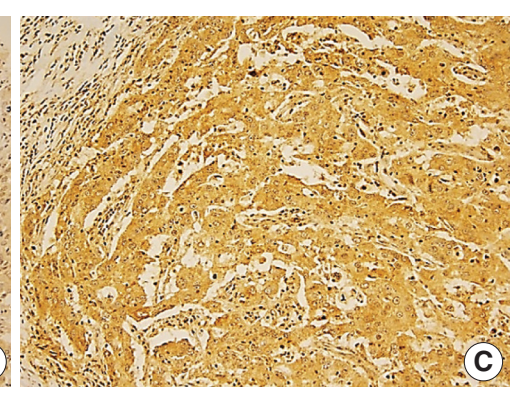

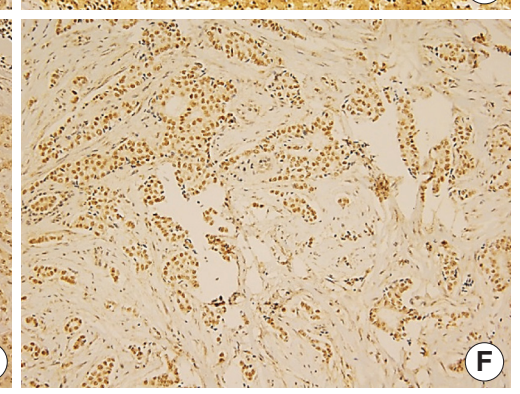

(D)
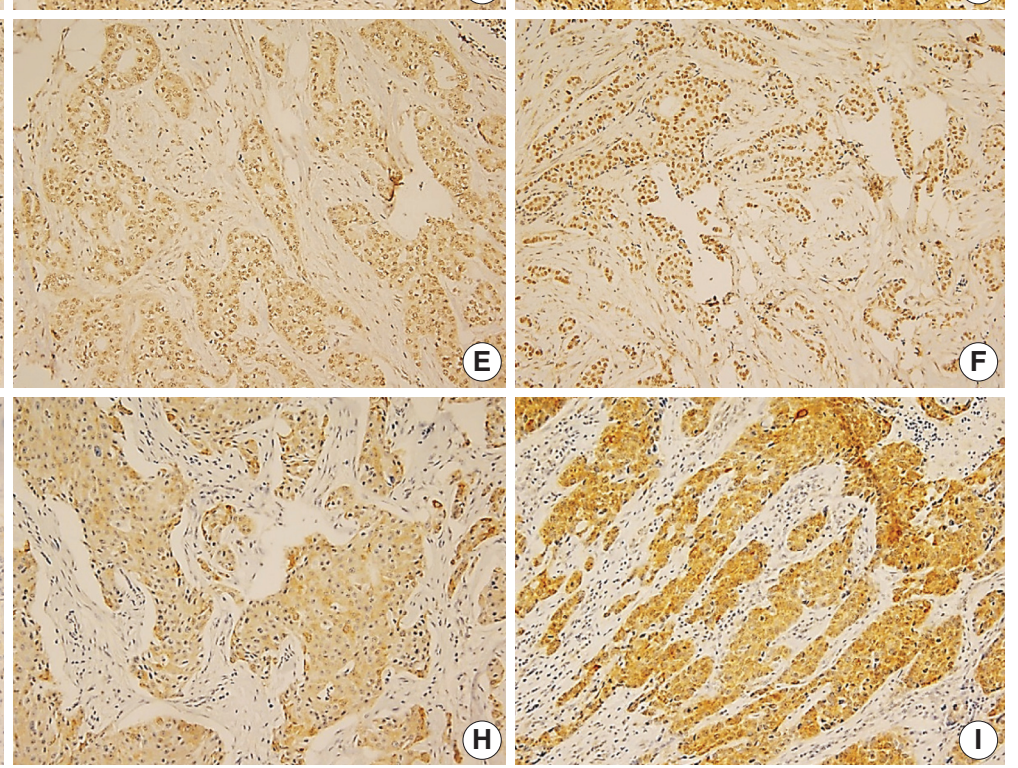

Fig. 1. Immunohistochemistry for CXC chemokine receptor type 4 (CXCR4) and CXC motif chemokine 12 (CXCL12). Representative immunohistochemistry images of cytoplasmic CXCR4 (A-C), nuclear CXCR4 (D-F), and CXCL12 (G-l) in order of staining intensity. CXCR4 and CXCL12 expression are mainly observed in tumor cells. CXCR4 shows cytoplasmic and nuclear staining, and CXCL12 shows cytoplasmic staining. 
and inflammatory cells, but staining was most prominent in the cytoplasm and nucleus of tumor cells (Fig. 1A-F). Staining for CXCL12 was mainly observed in tumor cell cytoplasm (Fig. 1GI). Expression of each marker was scored in tumor cells, and cytoplasmic and nuclear expression were separately assessed for CXCR4. Based on the median IHC score, high cytoplasmic CXCR4, high nuclear CXCR4, and high CXCL12 expression were defined as IHC score of $>7,>6$ and $>2$, and the tumors were classified into high cytoplasmic CXCR4, high nuclear CXCR4, and high CXCL12 groups in 194 (74.9\%), 115 (44.4\%), and 115 (48.3\%) cases, respectively.

\section{Clinicopathologic characteristics and expression of CXCR4 and CXCL12}

Correlations between clinicopathologic characteristics and expression of each marker are shown in Tables 2 and 3 . All patients were female and the median age at surgery was 48 years (range, 21 to 71 years). High cytoplasmic CXCR 4 expression was signif- icantly associated with younger age ( $\mathrm{p}=.008)$, higher histologic grade $(\mathrm{p}=.007)$, and lower pathologic stage $(\mathrm{p}=.045)$, but it was not related to tumor size or lymph node metastasis. On the other hand, high CXCL12 expression showed a significant correlation with larger tumor size $(\mathrm{p}=.045)$, positive lymph node metastasis $(\mathrm{p}=.005)$, and higher pathologic stage ( $\mathrm{p}=.017)$. Nuclear CXCR 4 expression was not associated with any of the clinicopathologic parameters studied. There was a significant difference in adjuvant chemotherapy regimen between high and low cytoplasmic CXCR 4 groups ( $\mathrm{p}=.009)$, but otherwise, no significant difference in adjuvant chemotherapy regimen or radiation therapy was observed between groups in other markers.

\section{Clinical outcome and pattern of recurrence after adjuvant chemotherapy}

The median follow-up time was 100 months (range, 1 to 141 months). During the follow-up period, the tumor recurred in 53 patients (18.7\%), and 17 patients (6.0\%) died. Locoregional and

Table 2. Clinicopathologic characteristics in relation to CXCR4 expression

\begin{tabular}{|c|c|c|c|c|c|c|c|}
\hline \multirow{2}{*}{ Variable } & \multirow{2}{*}{$\begin{array}{c}\text { Total } \\
(n=259)\end{array}$} & \multicolumn{3}{|c|}{ CXCR4 (cytoplasmic) } & \multicolumn{3}{|c|}{ CXCR4 (nuclear) } \\
\hline & & Low $(n=65)$ & High $(n=194)$ & $\mathrm{p}$-value & Low $(n=144)$ & High $(n=115)$ & $p$-value \\
\hline Age (yr) & & & & .008 & & & .524 \\
\hline$\leq 50$ & 152 & $29(19.1)$ & $123(80.9)$ & & $82(53.9)$ & $70(46.1)$ & \\
\hline$>50$ & 107 & 36 (33.6) & $71(66.4)$ & & $62(57.9)$ & 45 (42.1) & \\
\hline Histologic grade & & & & .007 & & & .310 \\
\hline I, || & 47 & $19(40.4)$ & $28(59.6)$ & & $23(48.9)$ & 24 (51.1) & \\
\hline III & 212 & $46(21.7)$ & $166(78.3)$ & & $121(57.1)$ & $91(42.9)$ & \\
\hline Size $(\mathrm{cm})$ & & & & $.131^{\mathrm{a}}$ & & & .957 \\
\hline$\leq 5$ & 243 & $58(23.9)$ & $185(76.1)$ & & $135(55.6)$ & $108(44.4)$ & \\
\hline$>5$ & 16 & $7(43.8)$ & $9(56.3)$ & & $9(56.3)$ & $7(43.8)$ & \\
\hline Lymph node metastasis & & & & .473 & & & .476 \\
\hline Negative & 165 & 39 (23.6) & $126(76.4)$ & & 89 (53.9) & $76(46.1)$ & \\
\hline Positive & 94 & $26(27.7)$ & 68 (72.3) & & 55 (58.5) & $39(41.5)$ & \\
\hline Stage & & & & .045 & & & .087 \\
\hline I, II & 216 & 49 (22.7) & $167(77.3)$ & & $115(53.2)$ & $101(46.8)$ & \\
\hline III & 43 & 16 (37.2) & $27(62.8)$ & & $29(67.4)$ & $14(32.6)$ & \\
\hline Histologic type & & & & .992 & & & .144 \\
\hline IDC & 239 & $60(25.1)$ & $179(74.9)$ & & $136(56.9)$ & $103(43.1)$ & \\
\hline Other ${ }^{b}$ & 20 & $5(25.0)$ & $15(75.0)$ & & $8(40.0)$ & $12(60.0)$ & \\
\hline Adjuvant chemotherapy regimen ${ }^{c}$ & & & & .009 & & & .538 \\
\hline Anthracycline-based & 149 & $27(18.1)$ & $122(81.9)$ & & $79(53.0)$ & $70(47.0)$ & \\
\hline Taxane-anthracycline-based & 49 & $14(28.6)$ & $35(71.4)$ & & $30(61.2)$ & $19(38.8)$ & \\
\hline $\mathrm{CMF}$ & 58 & $22(37.9)$ & $36(62.1)$ & & $34(58.6)$ & $24(41.4)$ & \\
\hline Radiation therapy ${ }^{d}$ & & & & .506 & & & .975 \\
\hline No & 84 & $23(27.4)$ & $61(72.6)$ & & $47(56.0)$ & $37(44.0)$ & \\
\hline Yes & 174 & $41(23.6)$ & $133(76.4)$ & & $97(55.7)$ & $77(44.3)$ & \\
\hline
\end{tabular}

Values are presented as number (\%).

CXCR4, CXC chemokine receptor type 4; IDC, Invasive ductal carcinoma; CMF, cyclophosphamide, methotrexate, and 5-fluorouracil.

aFisher exact test; ${ }^{I}$ nvvasive lobular carcinoma (2), mixed invasive ductal and lobular carcinoma (3), invasive papillary carcinoma (2), metaplastic carcinoma (8), medullary carcinoma (1), apocrine carcinoma (3), signet ring cell carcinoma (1); ‘3 missing values, unknown (2), change of regimen (1); d 1 missing value, unknown (1). 
Table 3. Clinicopathologic characteristics in relation to CXCL12 expression

\begin{tabular}{|c|c|c|c|c|}
\hline \multirow[b]{2}{*}{ Variable } & \multirow{2}{*}{$\begin{array}{c}\text { Total } \\
(\mathrm{n}=238)\end{array}$} & \multicolumn{3}{|c|}{ CXCL12 } \\
\hline & & $\begin{array}{c}\text { Low } \\
(n=123)\end{array}$ & $\begin{array}{c}\text { High } \\
(\mathrm{n}=115)\end{array}$ & $\mathrm{p}$-value \\
\hline Age (yr) & & & & .721 \\
\hline$\leq 50$ & 140 & $71(50.7)$ & $69(49.3)$ & \\
\hline$>50$ & 98 & $52(53.1)$ & $46(46.9)$ & \\
\hline Histologic grade & & & & .155 \\
\hline ।, II & 43 & $18(41.9)$ & $25(58.1)$ & \\
\hline III & 195 & $105(53.8)$ & $90(46.2)$ & \\
\hline Size (cm) & & & & .045 \\
\hline$\leq 5$ & 223 & $119(53.4)$ & $104(46.6)$ & \\
\hline$>5$ & 15 & $4(26.7)$ & $11(73.3)$ & \\
\hline Lymph node metastasis & & & & .005 \\
\hline Negative & 158 & $92(58.2)$ & $66(41.8)$ & \\
\hline Positive & 80 & $31(38.8)$ & $49(61.3)$ & \\
\hline Stage & & & & .017 \\
\hline I, II & 202 & $111(55.0)$ & $91(45.0)$ & \\
\hline III & 36 & $12(33.3)$ & $24(66.7)$ & \\
\hline Histologic type & & & & .931 \\
\hline IDC & 219 & $113(51.6)$ & $106(48.4)$ & \\
\hline Other ${ }^{a}$ & 19 & $10(52.6)$ & $9(47.4)$ & \\
\hline Adjuvant chemotherapy regime & & & & .117 \\
\hline Anthracycline-based & 144 & $82(56.9)$ & $62(43.1)$ & \\
\hline Taxane-anthracycline-based & 38 & $15(39.5)$ & $23(60.5)$ & \\
\hline $\mathrm{CMF}$ & 53 & $25(47.2)$ & $28(52.8)$ & \\
\hline Radiation therapy ${ }^{\circ}$ & & & & .408 \\
\hline No & 79 & $38(48.1)$ & $41(51.9)$ & \\
\hline Yes & 158 & $85(53.8)$ & $73(46.2)$ & \\
\hline
\end{tabular}

Values are presented as number (\%).

CXCL12, CXC motif chemokine 12; IDC, Invasive ductal carcinoma; CMF, cyclophosphamide, methotrexate, and 5 -fluorouracil.

anvasive lobular carcinoma (2), mixed invasive ductal and lobular carcinoma (2), invasive papillary carcinoma (2), metaplastic carcinoma (6), apocrine carcinoma (4), medullary carcinoma (1), signet ring cell carcinoma (1), clear cell carcinoma (1); b3 missing values, unknown (2), change of regimen (1); ${ }^{c} 1$ missing value, unknown (1). distant recurrence occurred in 18 (6.4\%) and 37 (13.1\%) patients, respectively. Distant recurrence was less frequent in the high cytoplasmic CXCR 4 group ( $\mathrm{p}=.006$ ), whereas nuclear CXCR4 and CXCL12 showed no significant association with any pattern of recurrence (Fig. 2A, B). Univariate logistic regression analysis revealed that high cytoplasmic CXCR 4 expression was associated with lower distant recurrence $(\mathrm{p}=.007)$. After multivariate analysis, high cytoplasmic CXCR 4 expression remained an independent variable for lower distant recurrence $(\mathrm{p}=.019)$ along with smaller tumor size $(\mathrm{p}=.042)$ and negative lymph node metastasis $(\mathrm{p}=.001)($ Table 4$)$.

\section{RFS and OS after adjuvant chemotherapy}

Kaplan-Meier curves for RFS and OS was plotted according to the expression of each marker. A significant difference in RFS was observed between high and low cytoplasmic CXCR 4 groups ( $\log$-rank $\mathrm{p}=.020)$, but the difference was not significant in OS (log-rank p=.076) (Fig. 3A, B). The 5-year RFS in high and low cytoplasmic CXCR 4 groups were $86.0 \%$ and $75.1 \%$, respectively. The 5-year OS in high and low cytoplasmic CXCR 4 groups were $96.0 \%$ and $90.7 \%$, respectively. No significant difference in survival was observed between groups in nuclear CXCR4 (logrank $\mathrm{p}=.637$ for RFS, $\mathrm{p}=.121$ for OS) and CXCL12 (log-rank $\mathrm{p}=.521$ for RFS, $\mathrm{p}=.538$ for OS). In univariate Cox regression analysis, high cytoplasmic CXCR 4 expression was associated with better RFS ( $\mathrm{p}=.022)$. Multivariate analysis revealed that cytoplasmic CXCR 4 expression ( $\mathrm{p}=.038$ ) and lymph node metastasis $(\mathrm{p}<.001)$ were independent factors of RFS (Table 5).
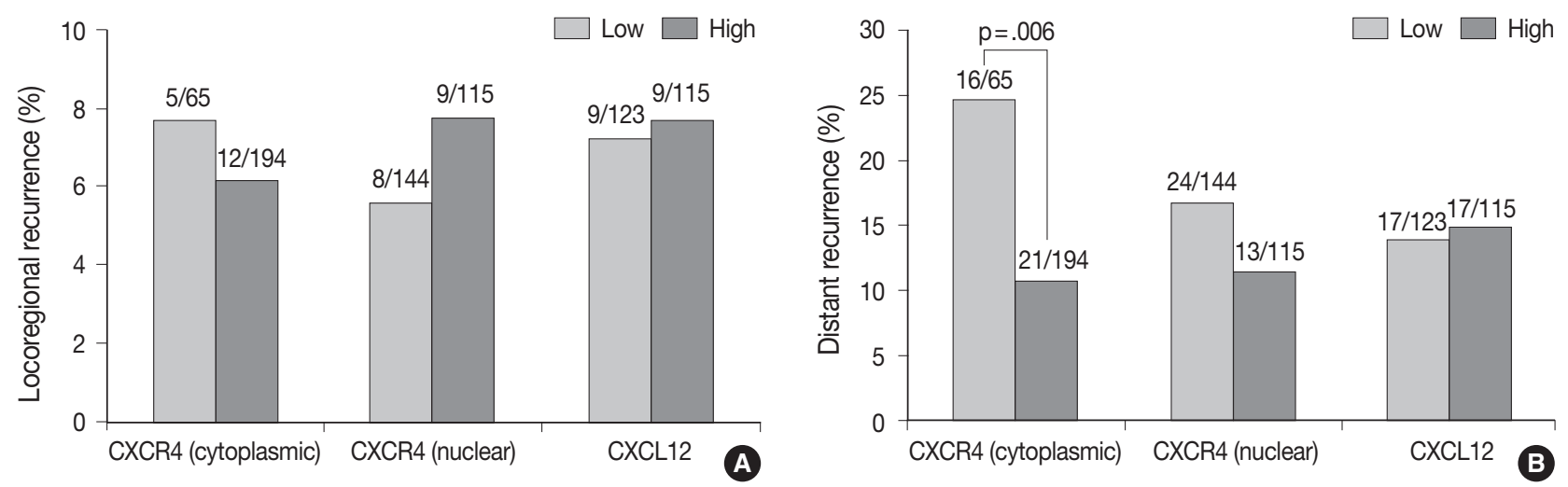

Fig. 2. The pattern of recurrence after adjuvant chemotherapy according to CXC chemokine receptor type 4 (CXCR4) and CXC motif chemokine 12 (CXCL12) expression. CXCR4 and CXCL12 expression do not show a significant association with locoregional recurrence (A), while high cytoplasmic CXCR4 expression is significantly associated with lower distant recurrence (B). Figures above each bar refer to the number of recurrences/the number of patients in each group. 


\section{DISCUSSION}

Expression of CXCR 4 is reported in various types of tumors, and its ligand CXCL12 is expressed widely in tumor and normal tissues by cancer cells, stromal cells, endothelial cells, and immune cells. ${ }^{3,21}$ Binding of CXCL12 to CXCR 4 activates multiple signaling pathways promoting tumor growth and metastasis, and CXCR4/ CXCL12 axis has a role in tumor cell-microenvironment interac-

Table 4. Logistic regression analysis for distant recurrence after adjuvant chemotherapy

\begin{tabular}{|c|c|c|c|c|c|c|}
\hline & \multicolumn{3}{|c|}{ Univariate analysis } & \multicolumn{3}{|c|}{ Multivariate analysis } \\
\hline & OR & $95 \% \mathrm{Cl}$ & $p$-value & OR & $95 \% \mathrm{Cl}$ & $\mathrm{p}$-value \\
\hline Age $>50 y r$ & 1.431 & $0.715-2.862$ & .311 & - & - & - \\
\hline Histologic grade III & 0.790 & $0.338-1.844$ & .585 & - & - & - \\
\hline Tumor size $>5 \mathrm{~cm}$ & 4.985 & $1.796-13.837$ & .002 & 3.231 & $1.046-9.985$ & .042 \\
\hline Lymph node metastasis & 4.462 & $2.153-9.246$ & $<.001$ & 3.491 & $1.630-7.478$ & .001 \\
\hline Radiation therapy & 1.029 & $0.492-2.152$ & .940 & - & - & - \\
\hline High CXCR4 (cytoplasmic) & 0.372 & $0.180-0.766$ & .007 & 0.400 & $0.186-0.860$ & .019 \\
\hline High CXCR4 (nuclear) & 0.637 & $0.309-1.315$ & .223 & - & - & - \\
\hline High CXCL12 & 1.082 & $0.523-2.236$ & .832 & - & - & - \\
\hline
\end{tabular}

OR, odds ratio; Cl, confidence interval; CXCR4, CXC chemokine receptor type 4; CXCL12, CXC motif chemokine 12.
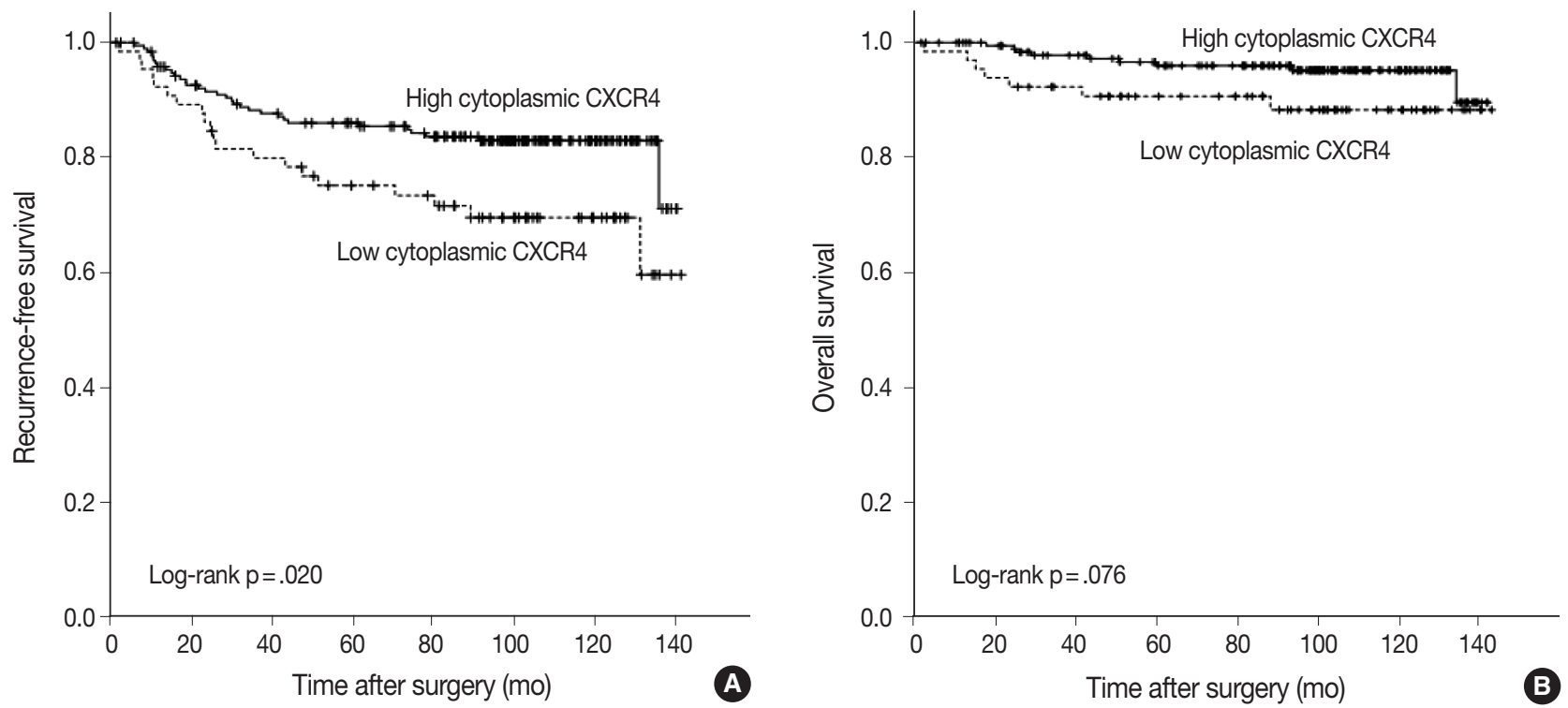

Fig. 3. Recurrence-free and overall survival after adjuvant chemotherapy according to cytoplasmic CXC chemokine receptor type 4 (CXCR4) expression. Recurrence-free survival is significantly better in the high cytoplasmic CXCR4 group (A), but the difference in overall survival is not significant between high and low cytoplasmic CXCR4 groups (B).

Table 5. Cox regression analysis for recurrence-free survival after adjuvant chemotherapy

\begin{tabular}{|c|c|c|c|c|c|c|}
\hline & \multicolumn{3}{|c|}{ Univariate analysis } & \multicolumn{3}{|c|}{ Multivariate analysis } \\
\hline & $\mathrm{HR}$ & $95 \% \mathrm{Cl}$ & $p$-value & $\mathrm{HR}$ & $95 \% \mathrm{Cl}$ & $p$-value \\
\hline Age $>50 y r$ & 0.959 & $0.554-1.660$ & .881 & - & - & - \\
\hline Histologic grade III & 0.909 & $0.468-1.766$ & .779 & - & - & - \\
\hline Tumor size $>5 \mathrm{~cm}$ & 3.116 & $1.467-6.617$ & .003 & - & - & - \\
\hline Lymph node metastasis & 3.298 & $1.908-5.702$ & $<.001$ & 3.005 & $1.724-5.237$ & $<.001$ \\
\hline Radiation therapy & 0.912 & $0.516-1.612$ & .751 & - & - & - \\
\hline High CXCR4 (cytoplasmic) & 0.521 & $0.298-0.912$ & .022 & 0.552 & $0.316-0.967$ & .038 \\
\hline High CXCR4 (nuclear) & 0.875 & $0.503-1.524$ & .637 & - & - & - \\
\hline High CXCL12 & 1.199 & $0.688-2.089$ & .522 & - & - & - \\
\hline
\end{tabular}

HR, hazard ratio; Cl, confidence interval; CXCR4, CXC chemokine receptor type 4; CXCL12, CXC motif chemokine 12. 
tion. ${ }^{21}$ In the present study, we evaluated the expression of CXCR 4 and CXCL12 on TMA constructed from TNBC tissue using the immunohistochemical method. Immunoreactivity for CXCR4 was observed primarily in the cytoplasm and nucleus of tumor cells, and CXCL12 was stained mostly in tumor cell cytoplasm. While CXCR 4 is a membrane-bound $\mathrm{G}$ protein-coupled receptor, it is rapidly internalized by binding of its ligand CXCL12, and cytoplasmic and nuclear expression of CXCR 4 was previously demonstrated in breast cancer by IHC. ${ }^{22-24}$ Our study also revealed that high cytoplasmic CXCR 4 expression was associated with higher histologic grade, and this finding was consistent with previous studies regarding breast cancer and TNBC. ${ }^{15,16,23-25}$ With respect to CXCL12 expression, Kobayashi et al. ${ }^{26}$ previously demonstrated that cytoplasmic-dominant CXCL12 immunoreactivity is associated with higher CXCL12 mRNA level in resected breast cancer.

Currently, there are a number of studies which evaluated the expression of CXCL12 in breast cancer. High CXCL12 expression correlated with better survival in most of these studies, but discrepant report exists. ${ }^{26-29}$ Ours is the first study to evaluate the expression of CXCL12 in TNBC subtype and showed that high CXCL12 expression was associated with known negative prognostic markers such as large tumor size, positive lymph node metastasis and higher stage in TNBC, although no significant difference in survival was observed between high and low CXCL12 groups. Loss of CXCL12 has been reported to have a role in distant metastasis of tumor cells, but overexpression of CXCL12 correlates with increased invasiveness, higher tumor grade and stage in several human cancers. ${ }^{30}$ The discordant result between the present and previous studies seems to suggest the different role of CXCL12 in TNBC subtype, and the different proportion of breast cancer subtypes in study population might have resulted in the discrepant results between previous studies.

In the evaluation of outcome and survival, the present study showed better survival compared with previous reports which studied the expression of CXCR 4 in TNBC. ${ }^{14-16}$ TNBC is known to be more sensitive to chemotherapy than other breast cancer subtypes, and since the study group was restricted to the patients who received adjuvant chemotherapy, it is likely that the effect of chemotherapy has contributed to the better survival observed in our study. ${ }^{2}$ The patient group with lower pathologic stage might have affected the survival as well. Our study also revealed that increased cytoplasmic expression of CXCR 4 was associated with a better prognosis in TNBC patients treated with adjuvant chemotherapy in terms of lower distant recurrence and better RFS. However, previous studies have reported high CXCR 4 expres- sion as a poor prognostic marker of TNBC, and antitumor effect of CXCR 4 inhibitors has been studied on breast cancer and shown efficacies in preclinical studies. ${ }^{12-16}$ On the other hand, Lefort $e t$ $\mathrm{all}^{31}$ recently reported that CXCR 4 inhibitors may not benefit TNBC patients and could even be detrimental in the study using patient-derived xenograft (PDX) model. In previous studies which evaluated the expression of CXCR4 in TNBC, Chu et al. ${ }^{14}$ used western blot analysis on 151 frozen tissue, and $\mathrm{Yu}$ et al. ${ }^{15}$ and Chen $e$ e $a l .{ }^{16}$ performed IHC on 148 and 75 FFPE samples, respectively. The difference in patient demographics, tissue preservation method, protein detection method, and scoring system may have caused the discordant results. However, this is the largest series of TNBC cases studied for the expression of CXCR 4 and CXCL12 to date, with 259 and 238 cases studied for CXCR 4 and CXCL12, respectively. Therefore, despite the limitation of retrospective study, we assumed that our data might have implications regarding the prognosis of TNBC.

The expression level of CXCR 4 and CXCL12 has been correlated with different hormone receptor and HER2 status in breast cancer. For example, high CXCL12 expression was associated with ER positivity in resected breast cancer, and CXCL12 expression was induced by estradiol treatment in ER-positive breast cancer cell lines. ${ }^{26,27}$ Salvucci et al. ${ }^{23}$ demonstrated that cytoplasmic CXCR4 expression was correlated with ER negativity, PR negativity, and HER2 expression, and Hassan et al. ${ }^{24}$ and Chen et al. ${ }^{16}$ reported that CXCR 4 expression level correlates with triplenegative status in breast cancer. Additionally, in the PDX model which recapitulated the stromal components of human breast cancer, CXCR 4 inhibition did not reduce tumor growth and even increased the distant metastasis of TNBC. ${ }^{31}$ Taken together, the difference in expression level and response to CXCR 4 inhibition suggests that CXCR4/CXCL12 axis may exert a different effect on metastasis and prognosis of TNBC compared with other breast cancer subtypes. Other components of tumor microenvironment may have a role in this phenomenon, but the exact mechanism needs to be investigated in future studies.

The study population in our study was restricted to the TNBC patients treated with adjuvant chemotherapy, and the result revealed that high cytoplasmic CXCR 4 expression is associated with lower distant recurrence and better RFS. Since adjuvant chemotherapy is indicated or recommended in most TNBC cases under current practice guideline, our result is clinically relevant and CXCR 4 expression might be useful in predicting outcome in TNBC patients after adjuvant chemotherapy. ${ }^{32}$ More importantly, we demonstrate for the first time that high CXCR 4 expression may be associated with better prognosis in TNBC patients, 
and suggest the possibility of the different mechanism underlying the metastasis and prognosis of TNBC.

This study has limitations. As previously noted, this was a retrospective study with known disadvantages. In addition, the patient population was heterogeneous in terms of chemotherapy regimen, and there was a significant difference in chemotherapy regimen between high and low cytoplasmic CXCR 4 expression groups. Use of taxane-anthracycline-based regimen was limited to node-positive cases, and since the patients received different chemotherapy based on nodal status, we could not compare the outcomes according to the chemotherapy regimen. Therefore, our results should be validated in prospective controlled cohort studies, and it would be beneficial to re-evaluate the prognostic significance of CXCR 4 in patients treated with the same chemotherapy regimen.

In conclusion, the present study indicates that high cytoplasmic expression of CXCR 4 may have a prognostic value in TNBC patients and predict lower distant recurrence and better RFS after adjuvant chemotherapy. To our knowledge, this is the first study to correlate high CXCR4 expression with better prognosis in TNBC, and the underlying mechanism needs to be explored in further studies.

\section{Conflicts of Interest}

No potential conflict of interest relevant to this article was reported.

\section{REFERENCES}

1. Boyle P. Triple-negative breast cancer: epidemiological considerations and recommendations. Ann Oncol 2012; 23 Suppl 6: vi7-12.

2. Bianchini G, Balko JM, Mayer IA, Sanders ME, Gianni L. Triplenegative breast cancer: challenges and opportunities of a heterogeneous disease. Nat Rev Clin Oncol 2016; 13: 674-90.

3. Balkwill F. Cancer and the chemokine network. Nat Rev Cancer 2004; 4: 540-50

4. Nagasawa T, Hirota S, Tachibana K, et al. Defects of B-cell lymphopoiesis and bone-marrow myelopoiesis in mice lacking the CXC chemokine PBSF/SDF-1. Nature 1996; 382: 635-8.

5. Baggiolini M. Chemokines and leukocyte traffic. Nature 1998; 392: 565-8.

6. Zou YR, Kottmann AH, Kuroda M, Taniuchi I, Littman DR. Function of the chemokine receptor CXCR4 in haematopoiesis and in cerebellar development. Nature 1998; 393: 595-9.

7. Domanska UM, Kruizinga RC, Nagengast WB, et al. A review on
CXCR4/CXCL12 axis in oncology: no place to hide. Eur J Cancer 2013; 49: 219-30

8. Orimo A, Gupta PB, Sgroi DC, et al. Stromal fibroblasts present in invasive human breast carcinomas promote tumor growth and angiogenesis through elevated SDF-1/CXCL12 secretion. Cell 2005; 121: $335-48$.

9. Muller A, Homey B, Soto H, et al. Involvement of chemokine receptors in breast cancer metastasis. Nature 2001; 410: 50-6.

10. Liu Y, Ji R, Li J, et al. Correlation effect of EGFR and CXCR4 and CCR7 chemokine receptors in predicting breast cancer metastasis and prognosis. J Exp Clin Cancer Res 2010; 29: 16.

11. Zhang Z, Ni C, Chen W, et al. Expression of CXCR4 and breast cancer prognosis: a systematic review and meta-analysis. BMC Cancer 2014; 14: 49.

12. Smith MC, Luker KE, Garbow JR, et al. CXCR4 regulates growth of both primary and metastatic breast cancer. Cancer Res 2004; 64: 8604-12.

13. Huang EH, Singh B, Cristofanilli M, et al. A CXCR4 antagonist CTCE-9908 inhibits primary tumor growth and metastasis of breast cancer. J Surg Res 2009; 155: 231-6.

14. Chu QD, Panu L, Holm NT, Li BD, Johnson LW, Zhang S. High chemokine receptor CXCR4 level in triple negative breast cancer specimens predicts poor clinical outcome. J Surg Res 2010; 159: 689-95.

15. Yu S, Wang X, Liu G, Zhu X, Chen Y. High level of CXCR4 in triplenegative breast cancer specimens associated with a poor clinical outcome. Acta Med Okayama 2013; 67: 369-75.

16. Chen HW, Du CW, Wei XL, Khoo US, Zhang GJ. Cytoplasmic CXCR4 high-expression exhibits distinct poor clinicopathological characteristics and predicts poor prognosis in triple-negative breast cancer. Curr Mol Med 2013; 13: 410-6.

17. Hammond ME, Hayes DF, Dowsett M, et al. American Society of Clinical Oncology/College of American Pathologists guideline recommendations for immunohistochemical testing of estrogen and progesterone receptors in breast cancer. J Clin Oncol 2010; 28: 2784-95.

18. Wolff AC, Hammond ME, Hicks DG, et al. Recommendations for human epidermal growth factor receptor 2 testing in breast cancer: American Society of Clinical Oncology/College of American Pathologists clinical practice guideline update. J Clin Oncol 2013; 31: 39974013.

19. Elston CW, Ellis IO. Pathological prognostic factors in breast cancer. I. The value of histological grade in breast cancer: experience from a large study with long-term follow-up. Histopathology 1991; 19: 403-10.

20. Hudis CA, Barlow WE, Costantino JP, et al. Proposal for standardized definitions for efficacy end points in adjuvant breast cancer trials: 
the STEEP system. J Clin Oncol 2007; 25: 2127-32.

21. Guo F, Wang Y, Liu J, Mok SC, Xue F, Zhang W. CXCL12/CXCR4: a symbiotic bridge linking cancer cells and their stromal neighbors in oncogenic communication networks. Oncogene 2016; 35: 816-26.

22. Haribabu B, Richardson RM, Fisher I, et al. Regulation of human chemokine receptors CXCR4. Role of phosphorylation in desensitization and internalization. J Biol Chem 1997; 272: 28726-31.

23. Salvucci O, Bouchard A, Baccarelli A, et al. The role of CXCR4 receptor expression in breast cancer: a large tissue microarray study. Breast Cancer Res Treat 2006; 97: 275-83.

24. Hassan S, Ferrario C, Saragovi U, et al. The influence of tumor-host interactions in the stromal cell-derived factor-1/CXCR4 ligand/receptor axis in determining metastatic risk in breast cancer. Am J Pathol 2009; 175: 66-73.

25. Guembarovski AL, Guembarovski RL, Hirata BK, et al. CXCL12 chemokine and CXCR4 receptor: association with susceptibility and prognostic markers in triple negative breast cancer. Mol Biol Rep 2018 Jun 20 [Epub]. https:/ /doi.org/10.1007/s11033-018-4215-7.

26. Kobayashi T, Tsuda H, Moriya T, et al. Expression pattern of stromal cell-derived factor-1 chemokine in invasive breast cancer is correlated with estrogen receptor status and patient prognosis. Breast Cancer
Res Treat 2010; 123: 733-45.

27. Mirisola V, Zuccarino A, Bachmeier BE, et al. CXCL12/SDF1 expression by breast cancers is an independent prognostic marker of diseasefree and overall survival. Eur J Cancer 2009; 45: 2579-87.

28. Yan M, Jene N, Byrne D, et al. Recruitment of regulatory T cells is correlated with hypoxia-induced CXCR4 expression, and is associated with poor prognosis in basal-like breast cancers. Breast Cancer Res 2011; 13: R47.

29. Kang H, Watkins G, Parr C, Douglas-Jones A, Mansel RE, Jiang WG. Stromal cell derived factor-1: its influence on invasiveness and migration of breast cancer cells in vitro, and its association with prognosis and survival in human breast cancer. Breast Cancer Res 2005; 7: R402-10.

30. Samarendra H, Jones K, Petrinic T, et al. A meta-analysis of CXCL12 expression for cancer prognosis. Br J Cancer 2017; 117: 124-35.

31. Lefort S, Thuleau A, Kieffer Y, et al. CXCR4 inhibitors could benefit to HER2 but not to triple-negative breast cancer patients. Oncogene 2017; 36: 1211-22.

32. Lebert JM, Lester R, Powell E, Seal M, McCarthy J. Advances in the systemic treatment of triple-negative breast cancer. Curr Oncol 2018; 25(Suppl 1): S142-S50. 